This item was submitted to Loughborough's Research Repository by the author.

Items in Figshare are protected by copyright, with all rights reserved, unless otherwise indicated.

\title{
Degradation mechanisms of bioresorbable polyesters. Part 2, Effects of initial molecular weight and residual monomer
}

\section{PLEASE CITE THE PUBLISHED VERSION}

https://doi.org/10.1016/j.actbio.2014.01.017

\section{PUBLISHER}

Elsevier (@ Acta Materialia Inc.)

\section{VERSION}

AM (Accepted Manuscript)

\section{PUBLISHER STATEMENT}

This work is made available according to the conditions of the Creative Commons Attribution-NonCommercialNoDerivatives 4.0 International (CC BY-NC-ND 4.0) licence. Full details of this licence are available at: https://creativecommons.org/licenses/by-nc-nd/4.0/

\section{LICENCE}

CC BY-NC-ND 4.0

\section{REPOSITORY RECORD}

Gleadall, Andy, Jingzhe Pan, Marc-Anton Kruft, and Minna Kellomaki. 2014. "Degradation Mechanisms of Bioresorbable Polyesters. Part 2, Effects of Initial Molecular Weight and Residual Monomer". Loughborough University. https://hdl.handle.net/2134/26776. 


\title{
Degradation mechanisms of bioresorbable polyesters. Part 2. Effects of initial molecular weight and residual monomer
}

\author{
Andrew Gleadall ${ }^{\mathrm{a}}$, Jingzhe Pan*a ${ }^{\mathrm{a}}$, and Marc-Anton Kruft ${ }^{\mathrm{b}}$ \\ ${ }^{a}$ Department of Engineering, University of Leicester, Leicester, LE1 7RH, UK \\ ${ }^{\mathrm{b}}$ Purac Biomaterials, P.O. Box 21, 4200 AA Gorinchem, The Netherlands \\ * Corresponding author. Tel: +44 (0)116 223 1092; Fax: +44 (0)116 252 2525; E-mail: \\ jp165@le.ac.uk;
}

The final publication is available at Elsevier via http://dx.doi.org/10.1016/j.actbio.2014.01.017

\begin{abstract}
The paper presents an understanding of how initial molecular weight and initial monomer fraction affect the degradation of bioresorbable polymers in terms of the underlying hydrolysis mechanisms. A mathematical model was used to analyse the effects of initial molecular weight for various hydrolysis mechanisms including noncatalytic random scission, autocatalytic random scission, noncatalytic end scission or autocatalytic end scission. Different behaviours were identified to relate initial molecular weight to the molecular weight half-life and to the time until the onset of mass loss. The behaviours were validated by fitting the model to experimental data for molecular weight reduction and mass loss of samples with different initial molecular weight. Several publications that consider initial molecular weight were reviewed. The effect of residual monomer on degradation was also analysed, and shown to accelerate the reduction of molecular weight and mass loss. An inverse square root law relationship was found between molecular weight half-life and initial monomer fraction for autocatalytic hydrolysis. The relationship was tested by fitting the model to experimental data with various residual monomer contents.
\end{abstract}

Key words: Biodegradable polymers, random scission, end scission, initial molecular weight, residual monomer

\section{Introduction}

Biodegradable polymers are regularly used in the medical field for applications such as sutures, fixation plates, screws, and drug delivery. The major benefit over orthopaedic permanent metal devices is that the polymers degrade over a period of months or years to become fully absorbed by the body therefore negating the need for repeat surgery for 
removal. It is important to understand the degradation and factors that affect it. In this study the effects of initial molecular weight and residual monomers are considered, neither of which are fully understood at present.

Initial molecular weight significantly affects degradation as has been shown by several experimental publications [1-6]. If the initial molecular weight of biodegradable polymers varies by an order of magnitude, so too does the number of polymer chains and therefore the number of scissions required to halve molecular weight. In addition, the ratio of end scission to random scission is likely to be higher for lower molecular weight samples which have a higher ratio of bonds at the chain ends. Mass loss may also be affected by initial molecular weight since more monomers will be produced if there is a greater number of chain ends, due to the greater rate of end scission. Experimental results in which samples of different initial molecular weights show the same trends should actually be interpreted as evidence for different rates of chain scission. The effect of initial molecular weight depends of the type of hydrolysis such as noncatalytic random scission, autocatalytic random scission, noncatalytic end scission or autocatalytic end scission. These hydrolysis mechanisms are discussed in detail in part 1 of this series of papers [7]. The model simulates bulk mass loss as the diffusion of monomers or oligomers out of the polymer. Significant mass loss due to surface erosion is not typically expected for the polymers considered in this study. In addition, substantial mass loss may occur when the polymer begins to break apart in the very late stages of degradation, by which time the model is no longer valid. The most reliable use of the model is to simulate the time at which significant mass loss begins. A monomer is produced by each end scission whereas oligomers are produced when a random scission occurs near a chain end. One aim of this paper is to understand the effects of initial molecular weight and relate them to the fundamental hydrolysis mechanisms. Simulations are conducted using a more detailed version of a previously developed mathematical model [8-11].

A polymer may contain residual monomer that remains from polymerisation or is thermally generated from processing techniques such as melt extrusion. The initial monomer content in biodegradable polymers has been shown to have an impact on the degradation trends [6, 1214]. In all experiments of which the authors are aware, the rate of degradation has been accelerated by an increased initial monomer content. The acceleration can be substantial. In the experiments of Paakinaho et al. [12] residual monomers accelerated the rate of degradation of inherent viscosity and strength by approximately one order of magnitude. The experimental data in the literature $[6,12-14]$ support the theory of autocatalysis in which the degradation is catalysed by the acid chain ends of residual monomers. The initial monomer is 
not expected to affect degradation that is purely noncatalytic in nature. In this study, the effect of residual monomer is analysed by simulations with the mathematical model detailed in part 1 [7].

Initial molecular weight and residual monomer were chosen for analysis because they can vary in the experimental literature by several orders of magnitude, have a significant effect on degradation, and are not fully understood. As a result of the lack of clear understanding, these factors are often not discussed in the analysis of experimental data. The simple trends identified in this work can be used to estimate the effects of both factors. The mathematical model is ideally suited for the analysis of initial molecular weight and initial monomer content because both factors are directly implemented in it. Initial molecular weight is almost always stated in experimental publications, so the findings of this study can be used when analysing historical experimental data. Although residual monomer is not always measured, it can be estimated based on polymer processing and has been measured more often in recent years.

The mathematical model is fitted to experimental data containing various weight fractions of initial monomer, and also to experimental data containing various initial molecular weights. The experimental data discussed in this study are for poly(lactide) or poly(glycolide) polymers because a large number of experimental publications consider these polymers in the literature. The fittings serve to demonstrate the ability of the model to consider both factors and to relate the degradation trends to the underlying hydrolysis mechanisms. The authors are not aware of any models for chain scission of biodegradable polymers that have included initial monomer content to date.

It should be noted that the mathematical model used here is more complicated than previous models and is not targeted at predicting degradation characteristics from initial material properties but rather its strength lies in its ability to: 1) identify the individual hydrolysis mechanisms that are prominent in experimental studies, and 2) consider the effect of various factors with regards to specific hydrolysis mechanisms. If the effect of each individual factor in degradation can be understood in the future, then this and similar mathematical models will have far greater predicative capabilities.

\section{The mathematical model}

The mathematical hydrolysis model is described in part 1 [7]. Eqs. (1 - 5) here are only repeated to help explain how initial molecular weight and residual monomer affect the model. 
All the model parameters and units are given in Table 1 for reference. The rate of random scission $R_{r s}$ is given by

$$
\frac{d R_{r s}}{d t}=k_{r 1} C_{e}+k_{r 2} C_{e}\left(\frac{C_{a c i d}}{1-X_{c}}\right)^{n}
$$

and the rate of end scission $R_{e s}$ is given by

$$
\frac{d R_{e s}}{d t}=k_{e 1} C_{\text {end }}+k_{e 2} C_{\text {end }}\left(\frac{C_{a c i d}}{1-X_{c}}\right)^{n}
$$

which differs from Eq. (1) in that the reactant is chain ends $C_{\text {end }}$ for end scission instead of amorphous ester bonds $C_{e}$ for random scission. Separate reaction constants are used for noncatalytic random scission $k_{r 1}$, autocatalytic random scission $k_{r 2}$, noncatalytic end scission $k_{e 1}$, and autocatalytic end scission $k_{e 2}$. Initial molecular weight affects the rate of end scission from the outset since for a lower initial molecular weight there are more initial chains $N_{\text {chains } 0}$ and therefore more chain ends as given by

$$
C_{\text {end }}=2 N_{\text {chain }}=2 N_{\text {chainso }}+2\left(R_{r s}-\left(R_{\text {ol }} / m\right)\right)
$$

in which the concentration of random scissions $R_{r s}$ and ester units in oligomers $R_{o l}$ are both zero at the start of degradation. The rate of end scission therefore increases linearly with the number of chains and inversely with initial molecular weight. The rate of random scission in Eq. (1) does not increase in the same manner because it is not directly dependent on the number of chain ends. The ratio of end scission to random scission therefore increases. If molecular weight reduction is due to random scission and mass loss is due to end scission as suggested in part 1 [7], mass loss may be expected to occur earlier for a lower initial molecular weight. A greater rate of end scission results in more monomers $C_{m}$ and therefore a greater concentration of acid catalyst $C_{a c i d}$ as given by

$$
C_{\text {acid }}=C_{m}+\left(C_{o l} / m\right) \text {. }
$$

This means the rate of autocatalytic hydrolysis increases, for both random and end scission, and the ratio of autocatalytic to noncatalytic scissions increases. Another major effect of increasing the value of $N_{\text {chains } 0}$, due to lower initial molecular weight, is that the relative increase of $N_{\text {chain }}$ due to each random scission is reduced. In other words, increasing the terms in brackets on the right hand side of Eq. (3) during degradation becomes less significant as $N_{\text {chains } 0}$ increases. This affects the degradation of number averaged molecular weight, calculated by 
$M_{n}=\frac{\left(C_{e}+\omega X_{c}\right) M_{0}}{N_{\text {chain }}}$,

which depends primarily on the increase of $N_{\text {chain }}$. For a lower initial molecular weight the effect of each random scission on $M_{n}$ is reduced. The balance between an increased rate of random scission and reduced relative effect of each random scission depends on the values of the reaction rate constants $k_{r 1}, k_{r 2}, k_{e 1}$, and $k_{e 2}$.

As the initial monomer content increases, the initial concentration of acid catalyst also increases since $C_{m}$ increases in Eq. (4). This results in two main effects: 1) the rate of autocatalytic chain scission increases, and so too therefore does the rate of $M_{n}$ reduction and ratio of autocatalytic to noncatalytic hydrolysis, and 2) the relative increase in $C_{\text {acid }}$ for the same number of scissions is reduced since $C_{m}$ has a larger initial value in Eq. (4). As a result of point 2, the rate of autocatalytic random scission does not increase to such a great extent during degradation. Point 1 may shift the molecular weight - time curve, as discussed in part 1 , from noncatalytic to autocatalytic in appearance. Point 2 may have the opposite effect. The balance between these effects depends on the reaction rate constants $k_{r 1}, k_{r 2}, k_{e 1}$, and $k_{e 2}$.

\section{The effect of initial molecular weight}

The effect of initial molecular weight on the degradation of molecular weight and mass loss is analysed for several different types of hydrolysis including noncatalytic random scission, autocatalytic random scission, noncatalytic end scission, and autocatalytic end scission. Molecular weight degradation is characterised by the molecular weight half-life. It is often the case that polymer samples show zero mass loss for a long period at the start of degradation [15-18]. An indication of the likely duration of this delay before mass loss onset is characterised here by the time taken for water-soluble monomers and oligomers to account for $10 \%$ of the total polymer weight. To simplify the results, the simulations are amorphous unless fitting experimental data for crystallinity. Similarly, diffusion of water-soluble small chains has been previously studied by Wang et al. [8] so is not considered here unless fitting experimental data for mass loss.

The model is also fitted to experimental data for various initial $M_{n}$. Model constants that are used for all simulations in this study, unless otherwise stated, are given in Table 2. As in part 1 [7], an extremely small value of initial monomer is included to begin autocatalytic reactions. This value is not varied in this section in the interest of simplicity but it should be 
noted that experimental values are likely to be much higher. The fittings to experimental data can still be achieved if a high concentration of initial monomer is used.

All parameters related to crystallinity are set to zero for amorphous simulations. 
Table 1. Parameters that are used in the mathematical model

\begin{tabular}{|c|c|c|}
\hline Model parameter description & Symbol & Units \\
\hline oligomer production rate parameter & $\alpha$ & no units \\
\hline oligomer production rate parameter & $\beta$ & no units \\
\hline initial concentration of ester bonds in all phases & $C_{e 0}$ & $\mathrm{~mol} \mathrm{~m}{ }^{-3}$ \\
\hline concentration of ester bonds in amorphous chains & $C_{e}$ & $\mathrm{~mol} \mathrm{~m}{ }^{-3}$ \\
\hline concentration of catalysing carboxylic acid end groups & $C_{\text {acid }}$ & $\mathrm{mol} \mathrm{m}{ }^{-3}$ \\
\hline concentration of long chain ends & $C_{\text {end }}$ & $\mathrm{mol} \mathrm{m} \mathrm{m}^{-3}$ \\
\hline oligomer concentration (after diffusion) & $C_{o l}$ & $\mathrm{~mol} \mathrm{~m} \mathrm{~m}^{-3}$ \\
\hline monomer concentration (after diffusion) & $C_{m}$ & $\mathrm{~mol} \mathrm{~m}^{-3}$ \\
\hline diffusion coefficient (amorphous phase) & $D_{0}$ & $m^{2}$ day $^{-1}$ \\
\hline diffusion coefficient (pores) & $D_{\text {pore }}$ & $m^{2}$ day $^{-1}$ \\
\hline noncatalytic random scission reaction rate constant & $k_{r 1}$ & day $^{-1}$ \\
\hline noncatalytic end scission reaction rate constant & $k_{e 1}$ & day $^{-1}$ \\
\hline autocatalytic random scission reaction rate constant & $k_{r 2}$ & {$\left[\mathrm{~mol}^{-1} \mathrm{~m}^{3}\right]^{0.5}$ day $^{-1}$} \\
\hline autocatalytic end scission reaction rate constant & $k_{e 2}$ & {$\left[\mathrm{~mol}^{-1} \mathrm{~m}^{3}\right]^{0.5} \mathrm{day}^{-1}$} \\
\hline average degree of polymerisation of oligomers & $m$ & no units \\
\hline molar mass & $M_{0}$ & $\mathrm{~g} \mathrm{~mol}^{-1}$ \\
\hline number averaged molecular weight & $M_{n}$ & $\mathrm{~g} \mathrm{~mol}^{-1}$ \\
\hline dissociation power of the acid end group & $n$ & no units \\
\hline original concentration of chains & $N_{\text {chainso }}$ & $\mathrm{mol} \mathrm{m}^{-3}$ \\
\hline current concentration of chains & $N_{\text {chain }}$ & $\mathrm{mol} \mathrm{m}^{-3}$ \\
\hline end scission concentration & $R_{e s}$ & $\mathrm{~mol} \mathrm{~m}{ }^{-3}$ \\
\hline random scission concentration & $R_{r s}$ & $\mathrm{~mol} \mathrm{~m}{ }^{-3}$ \\
\hline total scissions concentration & $R_{S}$ & $\mathrm{~mol} \mathrm{~m}{ }^{-3}$ \\
\hline oligomer concentration (produced) & $R_{o l}$ & $\mathrm{~mol} \mathrm{~m}{ }^{-3}$ \\
\hline monomer concentration (produced) & $R_{m}$ & $\mathrm{~mol} \mathrm{~m}{ }^{-3}$ \\
\hline inverse molar volume of crystalline phase & $\omega$ & $\mathrm{mol} \mathrm{m}^{-3}$ \\
\hline degree of crystallinity & $X_{c}$ & volume fraction \\
\hline extended degree of crystallinity & $X_{\text {ext }}$ & volume fraction \\
\hline maximum degree of crystallinity & $X_{\max }$ & volume fraction \\
\hline crystal nucleation probability & $p_{x}$ & no units \\
\hline crystallite volume & $V_{c}$ & $\mathrm{~m}^{3}$ \\
\hline Avogadro's constant & $\eta_{A}$ & $\mathrm{~mol}^{-1}$ \\
\hline
\end{tabular}


Table 2. Model constants and initial variable values used in simulations in this study

\begin{tabular}{lll}
\hline Constant & Units & Value \\
\hline$C_{e 0}$ & mol m & 17300 \\
$n$ & no units & 0.5 \\
$M_{0}$ & g mol $^{-1}$ & 72 \\
$\alpha$ & no units & 28 \\
$\beta$ & no units & 2 \\
$m$ & no units & 4 \\
\hline Variables & Units & Initial Values \\
\hline$R_{o l} R_{r s} R_{e s} R_{s} C_{o l}$ & mol m & 0 \\
$R_{m} C_{m}$ & mol m & $10^{-10} C_{e 0}$ \\
$C_{e}$ & mol m$^{-3}$ & $C_{e 0}-\left(C_{m}+\omega X_{c}\right)$ \\
\hline
\end{tabular}

\subsection{Simulations to identify the effect of initial molecular weight on molecular weight} reduction

Scenarios for the effect of initial $M_{n}$ on the molecular weight half-life for each individual hydrolysis mechanism are shown in Fig. 1. Each time initial molecular weight is halved the $M_{n}$ half-life is halved for end scission $\left(k_{r 1}=k_{r 2}=0\right)$, doubled for noncatalytic random scission $\left(k_{r 2}=k_{e 1}=k_{e 2}=0\right)$, and increased by $10-15 \%$ for autocatalytic random scission $\left(k_{r 1}=k_{e 1}=k_{e 2}=0\right)$. For noncatalytic random scission, samples of different initial molecular weight have the same rate of chain cleavage. However, polymers with higher initial $M_{n}$ have fewer chains so the effect of each random scission on molecular weight is greater and thus there is an inverse relationship between $M_{n}$ half-life and initial molecular weight. In autocatalytic random scission, this effect is offset by the fact that for a lower initial molecular weight more oligomers are produced, since more scissions are required to equally reduce $M_{n}$, which accelerate the rate of chain scission. For pure end scission the $M_{n}$ half-life increases linearly with initial $M_{n}$.

Experimental data by Raman et al. [2] and Pitt et al. [3] are included in the figure. The data points are arbitrarily normalised to suit the existing curves. The curve for combined autocatalytic and noncatalytic random scission $\left(k_{e 1}=k_{e 2}=0\right)$ has the reaction rate ratio $k_{r 2} / k_{r 1}=10$ and the curve for combined autocatalytic random and autocatalytic end scission $\left(k_{r 1}=k_{e 1}=0\right)$ has the reaction rate ratio $k_{e 2} / k_{r 2}=3000$. The same reaction rate ratios are used in the fittings of the model to the experimental data in the next section. The autocatalytic random scission curve may be interpreted to fit the data of Pitt et al. [3], as opposed to a combination of random and end scission, but mass loss data does not support this theory as discussed shortly. There is almost no dependence of $M_{n}$ half-life on initial molecular weight 
for a combination of autocatalytic random and autocatalytic end scission. The effect of increasing the rate of end scission at a lower initial $M_{n}$, and therefore the rate of autocatalytic random scission, offsets the effect of more random scissions being required to reduce normalised molecular weight.

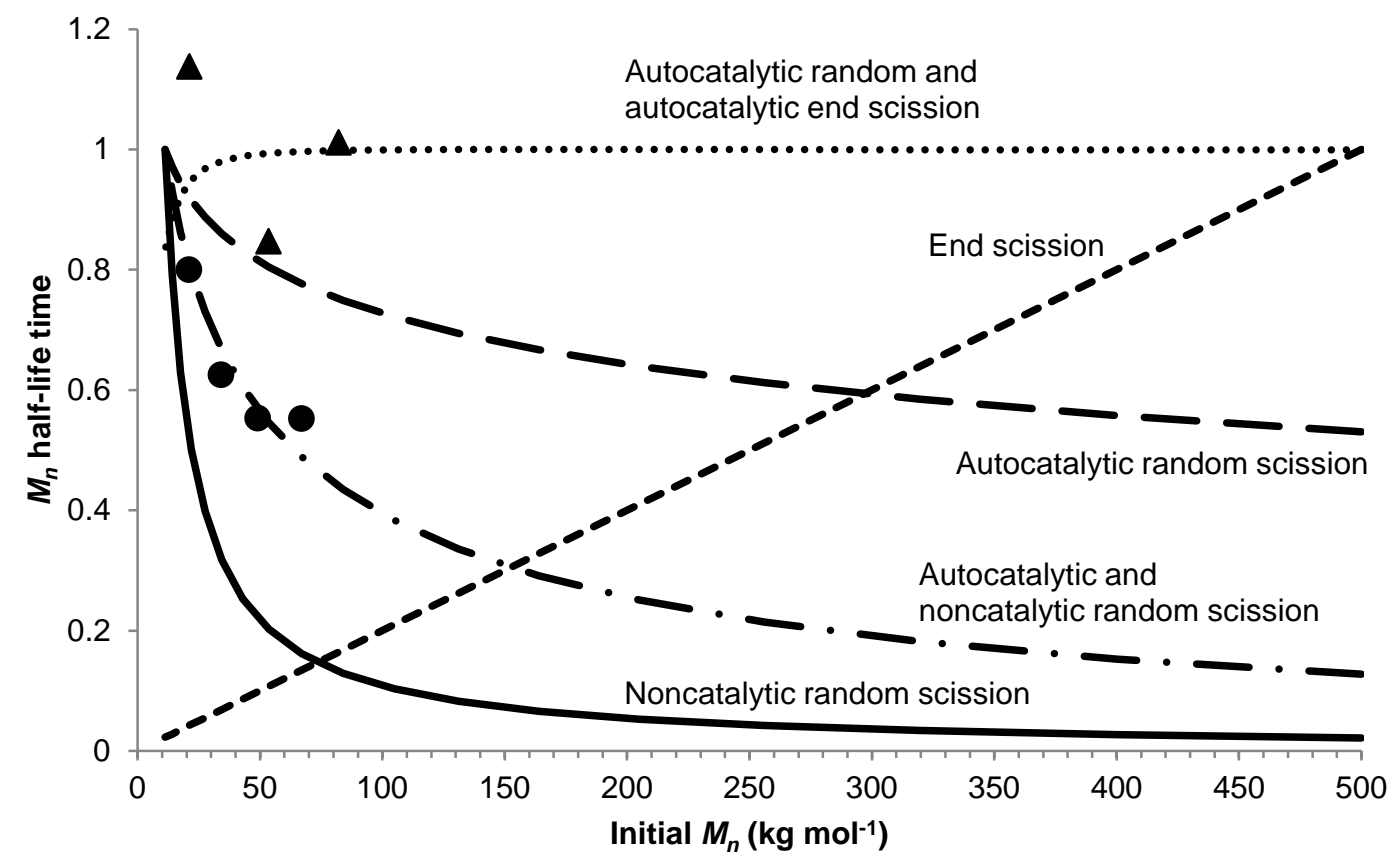

Fig. 1. Scenarios for $M_{n}$ half-life versus initial molecular weight for various theories of hydrolysis: noncatalytic random (solid); autocatalytic random (long dash); a combination of autocatalytic and noncatalytic random $\left(k_{r 2} / k_{r 1}=10\right.$, dash-dot); either autocatalytic or noncatalytic end (short dash); and a combination of autocatalytic random and autocatalytic end $\left(k_{e 2} / k_{r 2}=3000\right.$, dotted $)$. Experimental data for poly(lactide-co-glycolide) by Raman et al. [2] (circles) and poly(lactide) by Pitt et al. [3] (triangles) are included for reference.

\subsection{Simulations to identify the effect of initial molecular weight on mass loss}

Fig. 2 shows scenarios for the effect of initial molecular weight on the time taken for $10 \%$ of the total polymer weight to be water-soluble for various hydrolysis mechanisms. For end scission $\left(k_{r 1}=k_{r 2}=0\right)$, the rate of production of monomers is linearly related to the number of chain ends so the time taken for monomers to account for $10 \%$ of the polymer increases linearly with initial molecular weight. For random scission however $\left(k_{e 1}=k_{e 2}=0\right)$, initial molecular weight has negligible effect because the number of random scissions required to reduce $M_{n}$ from a typical high initial value (i.e. $3 \times 10^{5} \mathrm{~g} \mathrm{~mol}^{-1}$ ) to a typical low initial value 
(i.e. $3 \times 10^{4} \mathrm{~g} \mathrm{~mol}^{-1}$ ) is small compared to the number of scissions required to convert $10 \%$ of the polymer to oligomers. The trends are not affected by whether hydrolysis is noncatalytic or autocatalytic for either random or end scission. A curve for combined autocatalytic random and autocatalytic end scission $\left(k_{r 1}=k_{e 1}=0\right)$ is shown for the reaction rate ratio $k_{e 2} / k_{r 2}=3000$, which is the same as that used in the fitting of the model to the experimental data of Pitt et al. [3] in the next section. It could be argued that the experimental data points may fit to the end scission curve if they are normalised to an alternative value. However, the $M_{n}$ half-life data from the same experiments, shown on Fig. 1, do not resemble the curve for end scission in that figure.

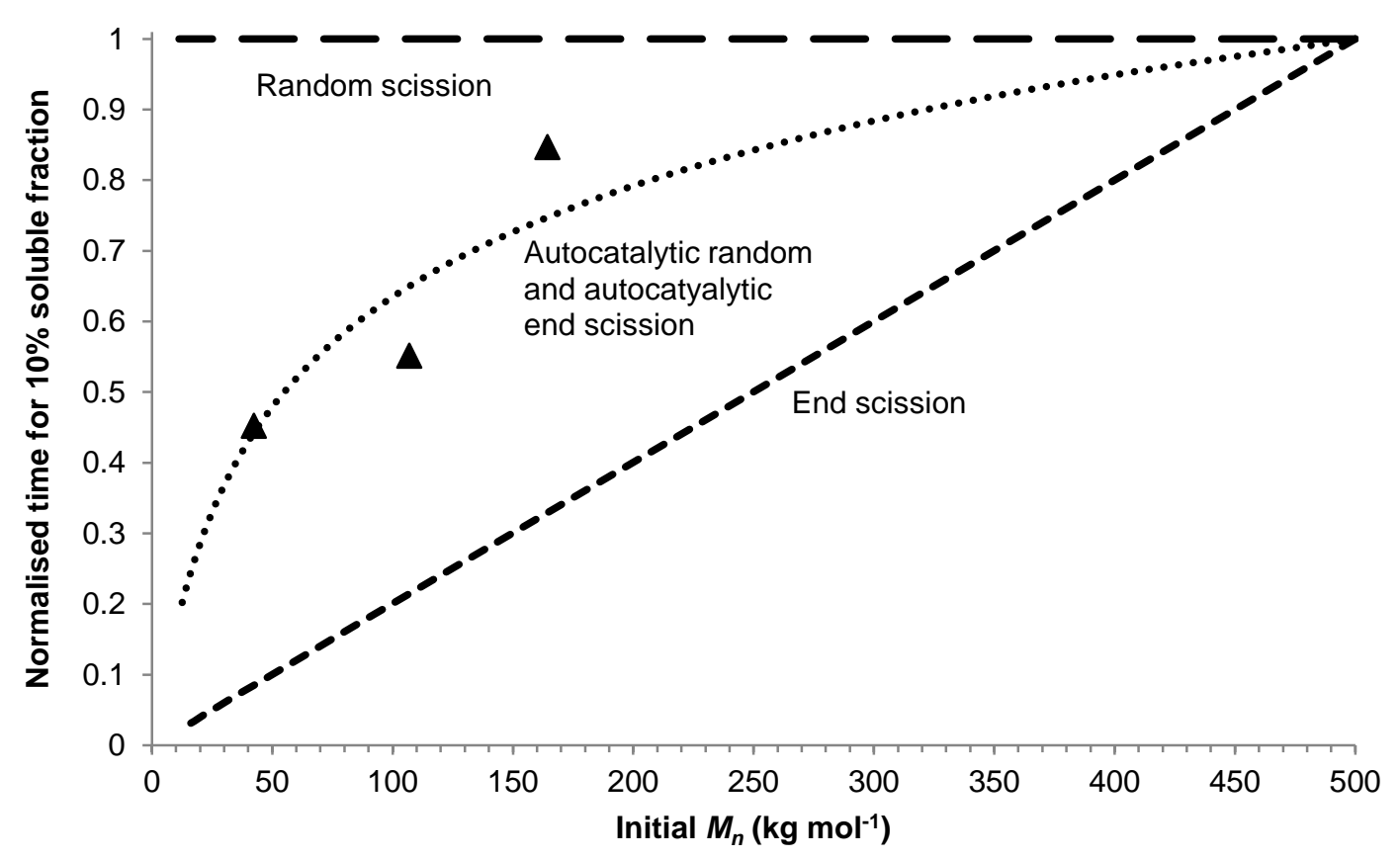

Fig. 2. Scenarios for the time until $10 \%$ of the polymer is water-soluble versus initial molecular weight is shown for various theories of hydrolysis: random scission (long dash), end scission (short dash), and an autocatalytic combination of both with a reaction rate ratio of $k_{e 2} / k_{r 2}=3000$ (dotted). Experimental data for poly(lactide) by Pitt et al. [3] (triangles) are included for reference.

\subsection{Fitting the model to experimental data}

Having identified the underlying mechanism, the model is used to fit the experimental data of Raman et al. [2] and Pitt et al. [3] which both consider the effect of initial molecular weight. The reaction rate ratios that are used in the fittings are also used for simulations in Fig. 1 and Fig. 2. 
Raman et al. [2] conducted degradation experiments on amorphous $50 \mu \mathrm{m}$ poly(DL-lactideco-glycolide) microspheres of various initial molecular weights in PBS pH 7.4 at $37^{\circ} \mathrm{C}$. The initial molecular weights were 67000, 49000, 34000, and $21000 \mathrm{~g} \mathrm{~mol}^{-1}$ for which the values of $N_{\text {chainso }}$ in the model are 16.8, 22.9, 33.0, and $53.4 \mathrm{~mol} \mathrm{~m}^{-3}$ respectively. The model setup is the same as the previous section and Table 2 except $M_{0}=65 \mathrm{~g} \mathrm{~mol}^{-1}$, which is the average molar mass of polylactic and polyglycolic acid. Fig. 3 shows that the model is able to fit the experimental data for a combination of noncatalytic and autocatalytic random scission as indicated by Fig. 1 . The reaction rates are $k_{r 1}=3 \times 10^{-5}$ day $^{-1}, k_{r 2}=3 \times 10^{-4}\left[\mathrm{~mol}^{-1} \mathrm{~m}^{3}\right]^{0.5} \mathrm{day}^{-1}$, and $k_{e 1}=k_{e 2}=0$. The same model parameters are used for all data sets except for $N_{\text {chains } 0 \text {, }}$ which represents initial $M_{n}$. This demonstrates the ability of the model to consider the effect of initial $M_{n}$. The fitting must use a combination of autocatalytic and noncatalytic random scission but cannot be improved by including end scission. For noncatalytic random scission alone $\left(k_{r 2}=k_{e 1}=k_{e 2}=0\right), M_{n}$ degrades too quickly for the samples with high initial $M_{n}$, and for autocatalytic random scission alone $\left(k_{r 1}=k_{e 1}=k_{e 2}=0\right), M_{n}$ degrades too slowly for the samples with high initial $M_{n}$.

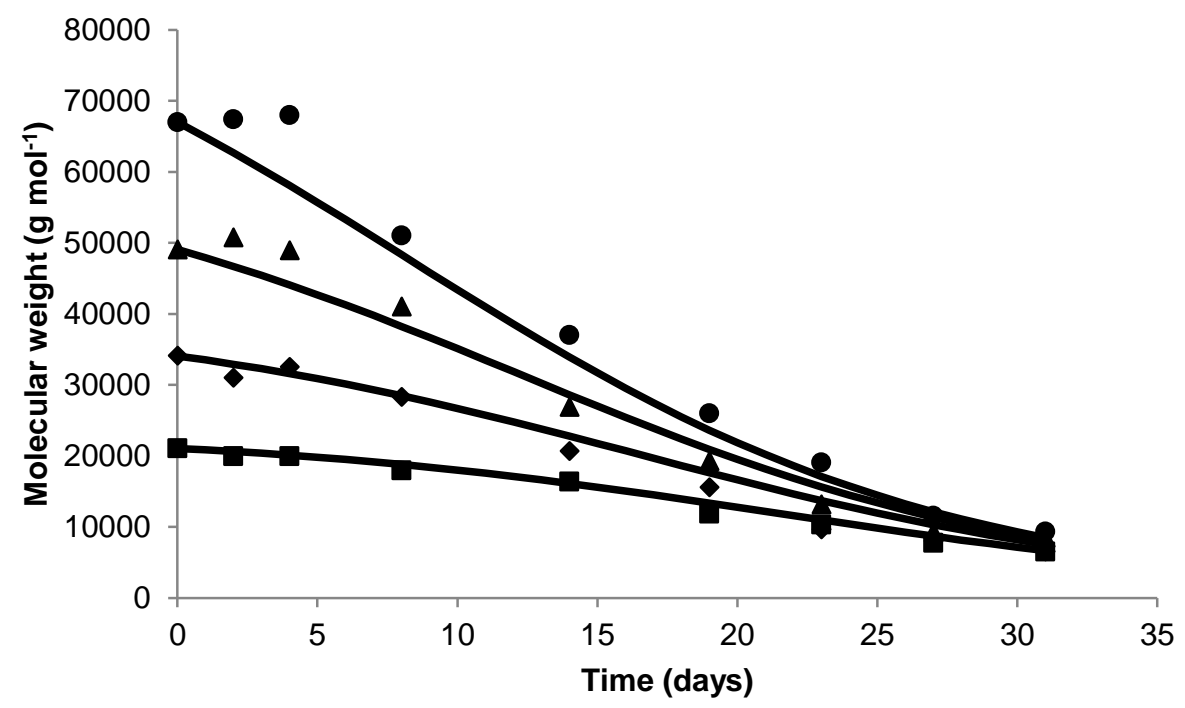

Fig. 3. Model fitting of molecular weight versus time for a combination of noncatalytic random and autocatalytic random scission. Discrete points represent experimental data [2] for various initial molecular weights and solid lines represent the model fitting.

Poly(DL-lactide) films of approximately $0.1 \mathrm{~mm}$ thickness were degraded in vivo by Pitt et al. [3]. The values of initial number averaged molecular weight were approximately 42000, 107000 , and $164000 \mathrm{~g} \mathrm{~mol}^{-1}$ for which the values of $N_{\text {chainso }}$ in the model are 29.3, 11.6, and 
$7.57 \mathrm{~mol} \mathrm{~m}^{-3}$ respectively. All other parameters are the same for all three data sets. A fitting of the mathematical model for a combination of autocatalytic end scission and autocatalytic random scission is shown in Fig. 4. The model setup is given in Table 2. Simulations are amorphous throughout so all crystallinity terms are set to zero. Diffusion is included in order model mass loss and the diffusion coefficients are $D_{0}=10^{-11} \mathrm{~m}^{2}$ week $\mathrm{k}^{-1}$ and $D_{\text {pore }}=10^{-7} \mathrm{~m}^{2}$ week $^{-1}$. Initial porosity is set to zero. The polymer film is represented by 200 finite difference nodes. Reaction rates are $k_{r 2}=1.25 \times 10^{-5}$ week $^{-1}, k_{e 2}=3.75 \times 10^{-2}\left[\mathrm{~mol}^{-1} \mathrm{~m}^{3}\right]^{0.5}$ week $^{-1}$, and $k_{r 1}=$ $k_{e 1}=0$. The experimental samples have very similar $M_{n}$ half-lives but mass loss occurs earlier for samples with lower initial $M_{n}$. The model is able to simulate these degradation behaviours by using a combination of end scission and random scission as would be identified by simple analysis of Fig. 1 and Fig. 2. Mass loss data for the model is not shown to a high percentage because the model is not designed for high mass loss, at which time parts of the polymer may break away and the water soluble chains may be able to diffuse through cracks in the polymer as opposed to through the polymer material.

The analyses on $M_{n}$ half-life and time to mass loss onset in Fig. 1 and Fig. 2 do not consider the shape of the molecular weight - time curves. As discussed in part 1 [7], the shape depends strongly on the type of hydrolysis. The fittings here must use the correct combination of hydrolysis mechanisms to 1) model the differences between degradation of samples with different initial molecular weights, and 2) model the shape of the molecular weight - time curves. The shapes of the molecular weight - time curves in Fig. 3 and Fig. 4 bear a good resemblance to the experimental data. The fittings for both sets of data are shown with normalised molecular weight in Fig. 5. This figure emphasises the trend seen in Fig. 1 that in the experiments of Pitt et al. [3] (Fig. 5 (b)) all samples degrade at a similar rate for normalised $M_{n}$, regardless of initial molecular weight, whereas in the experiments of Raman et al. [2] (Fig. 5 (a)) there is a reduced rate of $M_{n}$ degradation for samples with lower initial molecular weight. In the fittings to both sets of experimental data, the model finds a significantly increased rate of random chain scission in the samples of lower initial molecular weight. This is due to greater production of oligomers or monomers and is necessary in order to prevent the molecular weight of the high initial molecular weight samples reducing too quickly compared to the low initial molecular weight samples. For a combination of autocatalytic and noncatalytic hydrolysis, the samples of lower initial molecular weight therefore have a greater ratio of autocatalytic to noncatalytic hydrolysis, which can be seen in 
the slight shift of curves in Fig. 5. (a) from a noncatalytic shape to an autocatalytic shape, as detailed in part 1 [7].
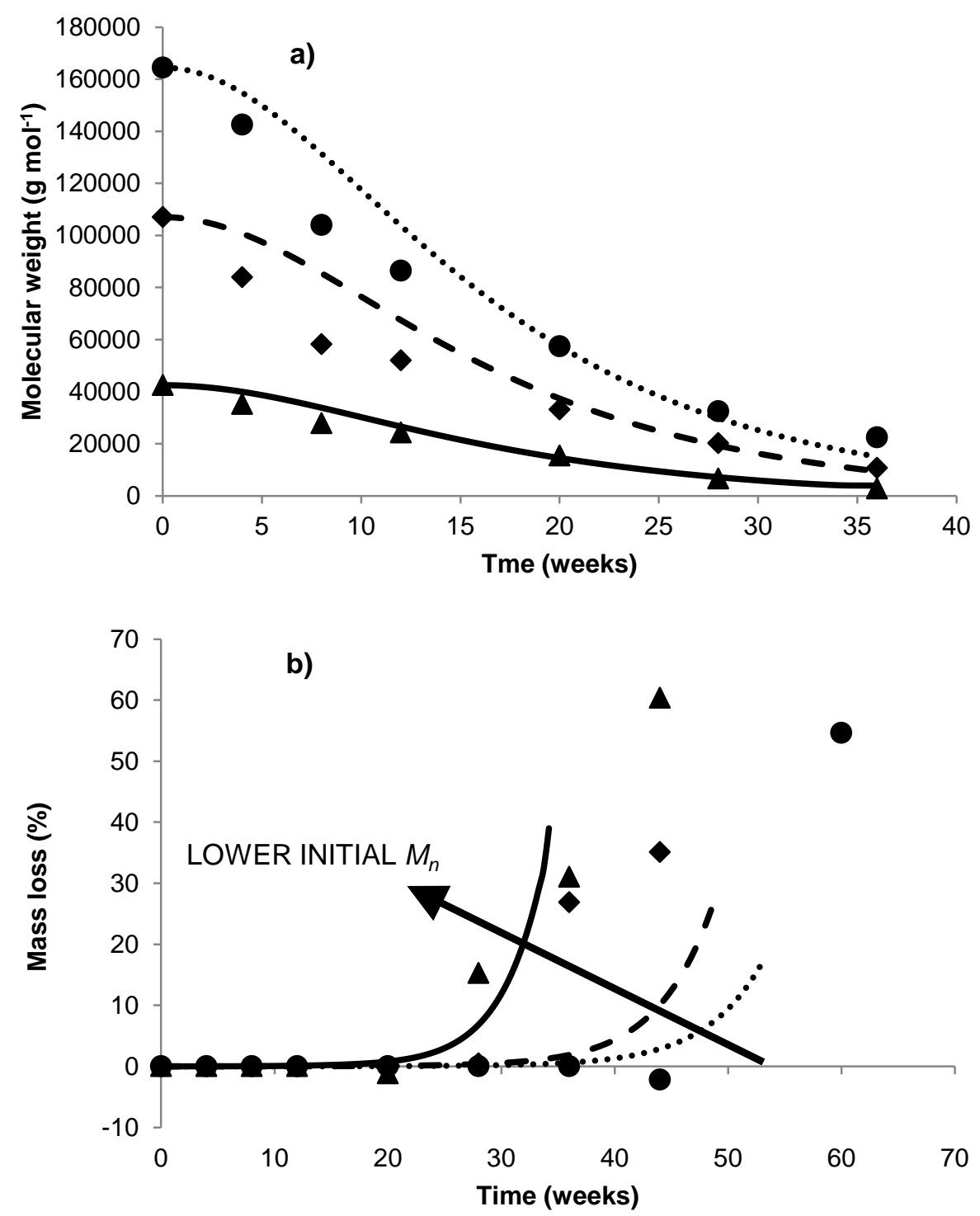

Fig. 4. Model fitting of a) molecular weight and b) mass loss versus time for a combination of autocatalytic random and autocatalytic end scission. Lines represent the model fitting and discrete points represent experimental data [3] for various initial $M_{n}$ : 42000 (triangle, solid line), 107000 (diamond, dashed line), and $164000 \mathrm{~g} \mathrm{~mol}^{-1}$ (circle, dotted line).

\subsection{Review of other publications for the effect of initial molecular weight}

The two sets of experimental data that have already been discussed [2, 3] demonstrated different effects of initial $M_{n}$. Other publications that also consider initial molecular weight are discussed here. 
Migliaresi et al. [1] studied the degradation of 3mm rods of amorphous poly(L-lactide) with initial molecular weights of 27000 and $177000 \mathrm{~g} \mathrm{~mol}^{-1}$ in Ringer solution at $37^{\circ} \mathrm{C}$. The molecular weight half-lives were approximately 100 days for both samples so Fig. 1 suggests
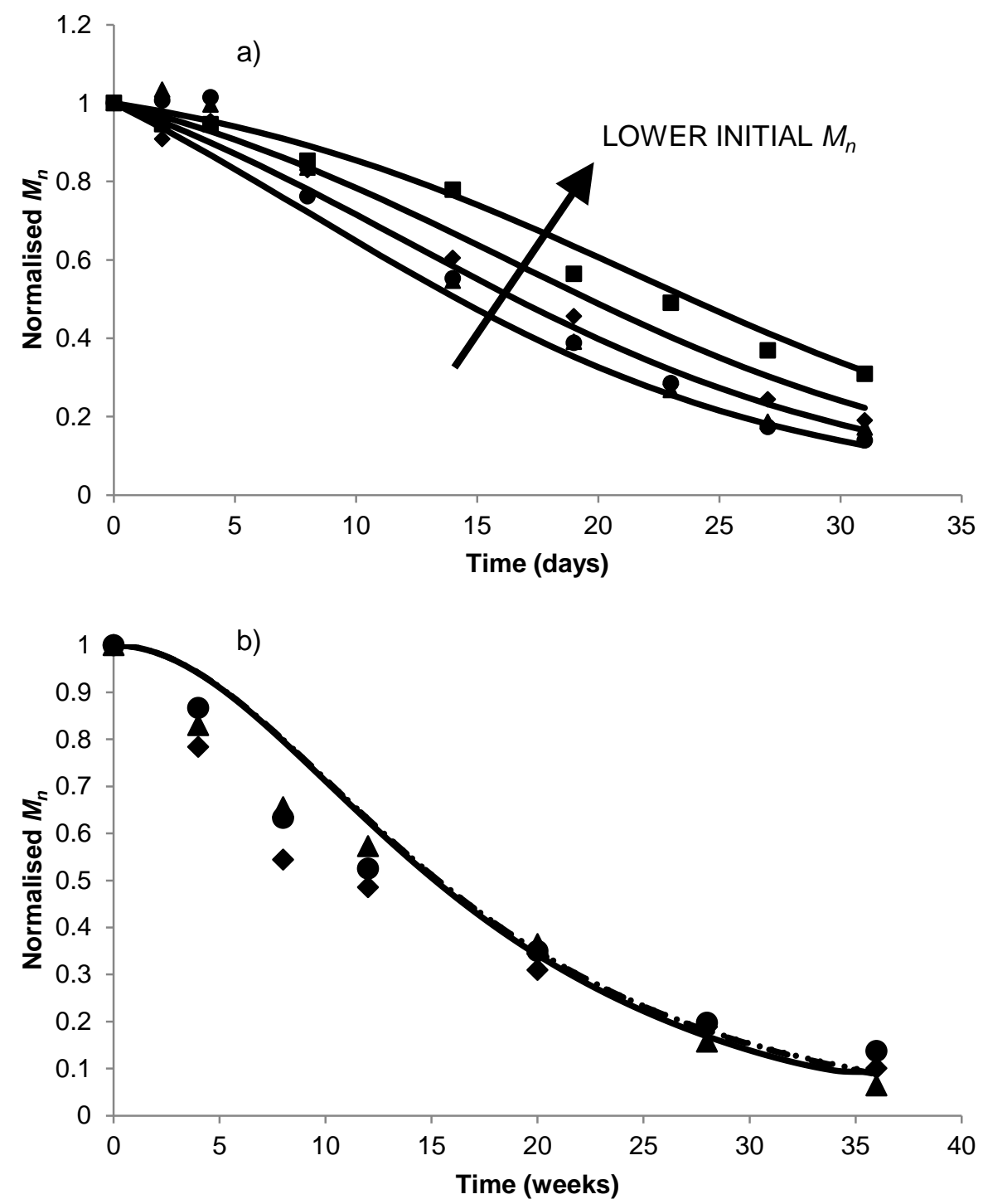

Fig. 5. Alternative presentation with normalised $M_{n}$ for a) the fitting shown in Fig. 3 and b) the fitting shown in Fig. 4. The shapes of discrete symbols and line types are the same as used previously.

a combination of autocatalytic random scission and autocatalytic end scission may give a good fitting of the model to experimental data.

Huttunen [6] compared the degradation of $4 \mathrm{~mm}$ poly(lactide) rods in $\mathrm{PBS} \mathrm{pH} 7.4$ at $37^{\circ} \mathrm{C}$ with initial inherent viscosities varying from 1.45 to $4.98 \mathrm{dl} \mathrm{g}^{-1}$. The experimental data is only given for the early stages of degradation but the inherent viscosity half-lives are similar for 
all samples, or perhaps slightly shorter for the samples with lower initial inherent viscosities. By comparison to Fig. 1, it can be seen that a good fitting may be achieved by a combination of autocatalytic random scission and autocatalytic end scission.

Witschi and Doelker [19] conducted experiments on poly(lactide-co-glycolide) 50:50 in PBS $\mathrm{pH} 7.4$ at $37^{\circ} \mathrm{C}$. The samples identified as RG-502 SD/WOW and RG-504 SD/WOW have the same copolymer ratio but different initial molecular weights of $\approx 6750$ and $\approx 13000 \mathrm{~g} \mathrm{~mol}^{-1}$ respectively. The lower molecular weight samples have similar or slightly longer $M_{n}$ halflives which suggests autocatalytic random scission is likely according to Fig. 1.

Grizzi, Li, and Vert [20, 21] conducted degradation experiments of 2mm thick Poly(DLlactide-co-L-lactide) plates in $\mathrm{PBS} \mathrm{pH} 7.4$ at $37^{\circ} \mathrm{C}$. The polymers had initial molecular weights of approximately 43000 and $65000 \mathrm{~g} \mathrm{~mol}^{-1}$. The time to the onset of mass loss is 5-9 weeks for the $43000 \mathrm{~g} \mathrm{~mol}^{-1}$ sample and $\approx 7$ weeks for the $65000 \mathrm{~g} \mathrm{~mol}^{-1}$ experiment. Given that the onset of mass loss occurs at similar times for both samples, Fig. 2 suggests that random scission without end scission is likely. Molecular weight data for the $65000 \mathrm{~g} \mathrm{~mol}^{-1}$ sample is too infrequent for comparison to Fig. 1.

Pistner et al. [4] carried out degradation experiments of $2 \mathrm{~mm}$ thick amorphous poly(Llactide) rods in vivo with initial molecular weights of 132000 and $197000 \mathrm{~g} \mathrm{~mol}^{-1}$. There is an approximately inverse relationship between $M_{n}$ half-life and initial molecular weight, which suggests noncatalytic random scission is dominant according to Fig. 1. Both samples begin to demonstrate mass loss at 42.5 weeks which suggests random scission is responsible for mass loss according to Fig. 2.

Hyon et al. [14] conducted experiments for the degradation of poly(DL-lactide) with initial molecular weights of 7000, 12000, and $43000 \mathrm{~g} \mathrm{~mol}^{-1}$. The samples were manufactured via different techniques and were expected to have varying concentrations of residual monomer which significantly affects degradation. Caution must therefore be taken when analysing the effect of initial molecular weight. The increased rate of degradation for lower molecular weight samples was attributed to an increased initial concentration of residual monomers. The effect of residual monomer is discussed in the next section of this paper, the findings of which agree with that theory. In the experiments, samples with lower initial $M_{n}$ demonstrated shorter $M_{n}$ half-lives and more accelerated mass loss. These trends are expected for a combination of autocatalytic random scission and autocatalytic end scission according to Fig. 1 and Fig. 2. It may be the case that both initial molecular weight and residual monomer affected the results in a similar manner. 
In the above analysis, the hydrolysis mechanisms suggested by comparison of experimental data to Fig. 1 and Fig. 2 agree with those suggested in part 1 [7]. This supports the trends identified in Fig. 1 and Fig. 2 along with ability of the model to consider the effect of initial molecular weight. It also supports the findings in part 1 [7] that random scission is responsible for the reduction of molecular weight and that autocatalytic hydrolysis may be more prevalent in degradation experiments than noncatalytic hydrolysis. One set of experiments that do not fit the expected trends predicted by the model are those of Park [5] which consider $10 \mu \mathrm{m}$ microspheres of poly(DL-lactide). The degradation rate of a high molecular weight sample was negligible compared to that of a low molecular weight sample. However, the values of initial molecular weight (900-1300 $\left.\mathrm{g} \mathrm{mol}^{-1}\right)$ are orders of magnitude lower than typical experimental poly(lactide) which resulted in one sample being beneath the glass transition temperature. The samples also contained high quantities of low molecular weight chains, which may have significantly affected hydrolysis in a similar manner to that discussed in the next section for residual monomers.

\section{The effect of residual monomers}

Residual monomer significantly affects degradation if autocatalytic hydrolysis is assumed to occur. Noncatalytic hydrolysis will by definition be unaffected. The model is used here to predict the effect of initial monomer content on the molecular weight half-life for autocatalytic hydrolysis. The model is also fitted to experimental data to demonstrate the ability of the model to consider residual monomer.

The effect of initial monomer content on the $M_{n}$ half-life is shown in Fig. 6 for autocatalytic random scission, autocatalytic end scission, and a combination of both with reaction rate ratios $k_{e 2} / k_{r 2}=10,10^{2}, 3 \times 10^{2}$, and $10^{3}$. The model setup is that same as that given in Table 2 except the initial monomer fraction varies from $C_{m}=R_{m}=10^{-1} C_{e 0}$ to $10^{-7} C_{e 0}$. The noncatalytic reaction rates are set to zero $\left(k_{r 1}=k_{e 1}=0\right)$ and $N_{\text {chains } 0}=42.4 \mathrm{~mol} \mathrm{~m}^{-3}$. The simulation is assumed to be amorphous throughout with no diffusion so all model parameters related to crystallinity and diffusion are set to zero. If molecular weight reduces due to random scission (as is the case in curves A-E in Fig. 6) there is an inverse square root law relationship between $M_{n}$ half-life and initial monomer fraction above a critical monomer content which depends on the ratio of end scission to random scission. This relationship can be derived analytically from the mathematical model. Experimental data for the inherent viscosity half-life of poly(lactide-co-glycolide) with various initial monomer contents [12] is 
included in the figure and can be seen to follow the predicted trend for autocatalytic random scission and autocatalytic end scission until the initial monomer content is increased above $2 \%$. The reaction rate ratio for the dotted line is the same at that used in the fitting of the model to the experimental data for molecular weight, crystallinity and mass loss later in this section $\left(k_{e 2} / k_{r 2}=3 \times 10^{2}\right)$. It can be seen to fit through the experimental data points well. The model appears to be suitable for initial monomer contents $<3 \%$, a range in which the expected initial monomer contents due typical processing techniques fall within. The cause of the 2 samples with highest initial monomer contents having lower than predicted $M_{n}$ half-lives cannot be explained by the model. It may be due to factors that are out of scope for the model such as changes in the polymer structure or the development of monomer-filled pores, as found in a previous study [14]. The mathematical model assumes that monomers are evenly distributed amongst the amorphous phase.

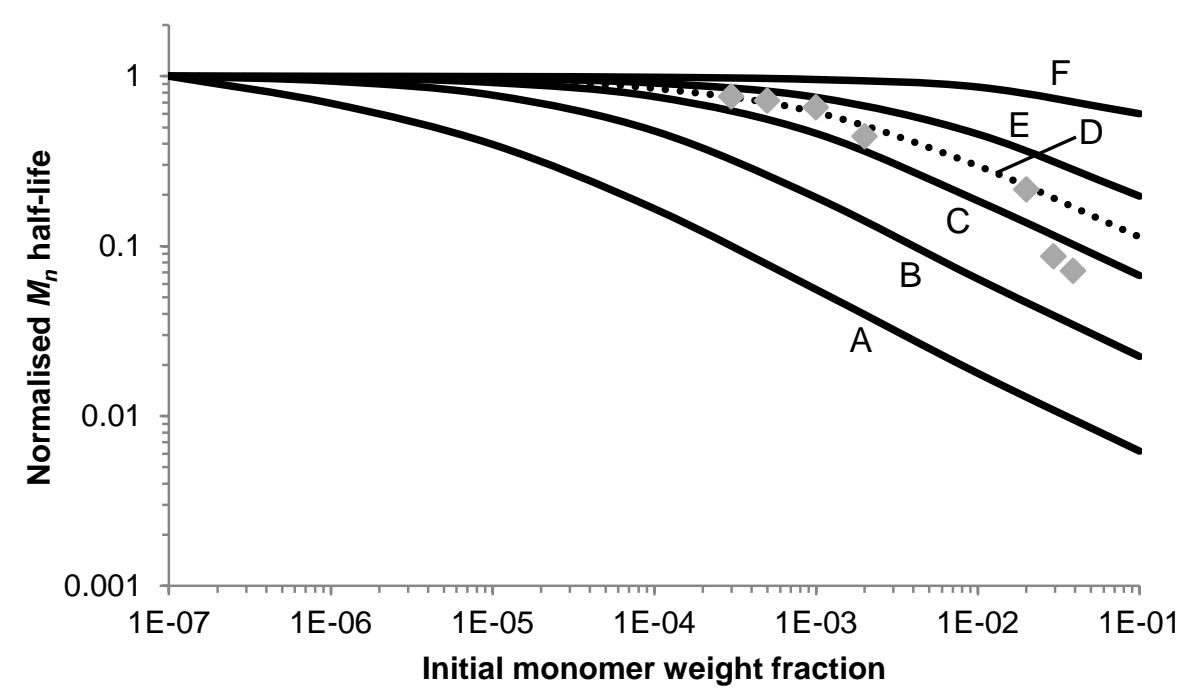

Fig. 6. Time to halve molecular weight versus initial monomer weight fraction for autocatalytic hydrolysis varying from random scission (A) to end scission (F). The end:random scission rate ratios $\left(k_{e 2} / k_{r 2}\right)$ for combined simulations are 10 (B), 100 (C), 300 (D), and 1000 (E). Discrete points are experimental data [12].

To test the ability of the mathematical model to incorporate initial monomer fraction, it is fitted to the experimental data of Paakinaho et al. [12] for inherent viscosity, crystallinity, and mass loss. In the experiments, $1.6 \mathrm{~mm}$ poly(lactide-co-glycolide) rods containing different fractions of initial monomer were degraded in $37^{\circ} \mathrm{C}$ Sörensen buffer solution. The initial monomer contents are $0.03 \%, 0.05 \%, 0.1 \%$, and $0.2 \%$, for which initial crystallinity $X_{c}=$ 
0.029, 0.067, 0.035, and 0.033 and $N_{\text {chains } 0}=42.4,46.9,52.4$, and 46.9 respectively. These values are derived from experimental measurements. The values of $N_{\text {chainso }}$ represent initial molecular weights of 2.94, 2.66, 2.38, and $2.66 \times 10^{4} \mathrm{~g} \mathrm{~mol}^{-1}$. These values are estimated through a linear relationship to initial inherent viscosity of $M_{n}=27900 \mathrm{IV}-6890$ in which the units of $M_{n}$ and IV are $\mathrm{g} \mathrm{mol}^{-1}$ and $\mathrm{dL} \mathrm{g}^{-1}$. It is the linear best fit for 22 data points of poly(L/D lactide) 96/4 with IV $<4 \mathrm{dL} \mathrm{g}^{-1}$ [13, 22]. The values of all other model parameters do not vary between the data sets and are given in Table 2 and Table 3 except $C_{m}=R_{m}=C_{e 0}$ $\mathrm{x} 0.003,0.005,0.01$, and $0.02 \mathrm{~mol} \mathrm{~m}^{-3}$ to reflect residual monomer and a value of $n=0.67$ gives the best fitting. The initial values of porosity and extended crystallinity are zero. There are 200 finite difference nodes to represent the rod diameter. The model is only being fitted to the four samples in which the monomer was produced during processing and for which mass loss was measured. In the other three samples, the monomer was added manually and may have a slightly different effect on degradation if, for example, monomers-filled pores develop as previously seen [14]. In the fitting, the reduction of normalised molecular weight (mathematical model) is compared to normalised inherent viscosity (experimental data) since molecular weight was not measured. The model is able to fit the data as can be seen in the fitting in Fig. 7. That the same set of model parameters is used for all samples demonstrates the ability of the model to simulate the effect of initial monomer. In the fitting, the noncatalytic reaction rates can be set to zero which suggests that chain cleavage due to noncatalytic hydrolysis is negligible compared to that of autocatalytic hydrolysis. This is to be expected since the initial monomers greatly increase the rate of autocatalytic scission, as can be seen in Fig. 6. in the effect of initial monomer on the molecular weight half-life. The fittings of $M_{n}$ to inherent viscosity are shown together in Fig. 8. The experimental data for the 2-4\% initial monomer samples, to which the model is not fitted, are also included for reference. As the initial monomer weight fraction increases, the catalysing carboxylic chain ends that are produced during hydrolysis make up a smaller fraction of the total concentration of catalyst. As a result, the catalyst, and therefore the rate of chain scission, does not increase to such an extent during degradation and the molecular weight - time curves shift from autocatalytic (degradation rate accelerating) towards noncatalytic in appearance, as shown in Fig. 1. in part 1 [7]. Although the molecular weight - time curves shift towards noncatalytic in appearance, the results still support a theory of autocatalytic hydrolysis because the monomers catalyse the hydrolysis. It is simply the case that the negligible increase in catalyst during degradation results in a similar trend to noncatalytic hydrolysis. One remarkable interpretation of this could be that many experimental publications which appear to 
demonstrate noncatalytic hydrolysis may have actually undergone autocatalytic hydrolysis but contained high residual monomer. Paakinaho et al. [12] even found the concentration of monomer catalyst to decrease during degradation. This is why the rate of $M_{n}$ reduction for the 4\% sample decelerates to an even greater extent than the curve for noncatalytic random scission in Fig. 1. in part 1 [7]. In the experiments of Paakinaho et al. [12], the monomer diffused out of the $4 \%$ sample at a much greater rate than the $2 \%$ sample. Therefore it is plausible that the monomer does not diffuse out rapidly in samples with $0.03-0.2 \%$ residual monomer. It should be noted that if the residual monomer catalyst is assumed to diffuse out of the polymer in the first few weeks there may still be long term effects because the chain scissions in the first few weeks lead to more chains ends and therefore a greater rate of end scission throughout the duration of degradation. 

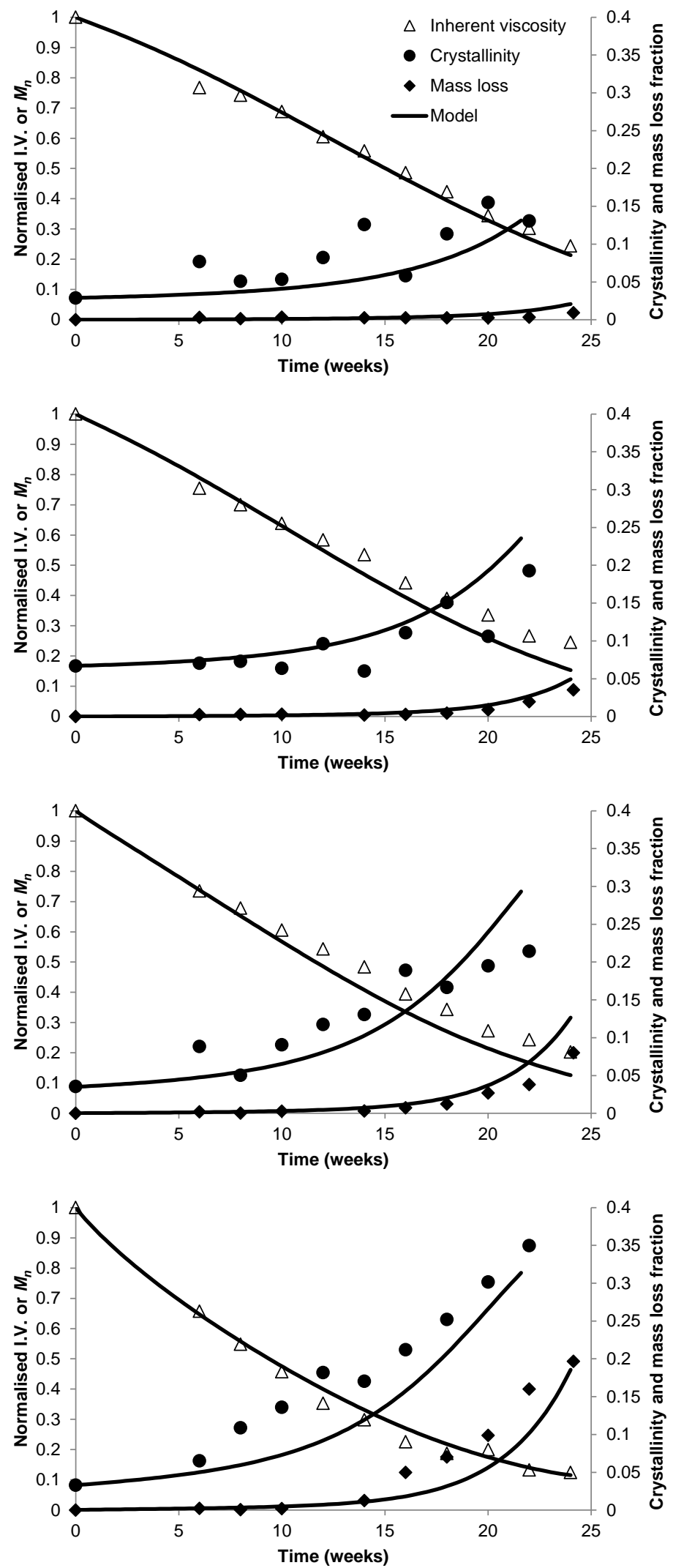

Fig. 7. Model fitting to the experimental data [12] with an assumption of a combination of autocatalytic random scission and autocatalytic end scission. Initial monomer contents are a) $0.03 \%$, b) $0.05 \%$, c) $0.1 \%$, and d) $0.2 \%$. 


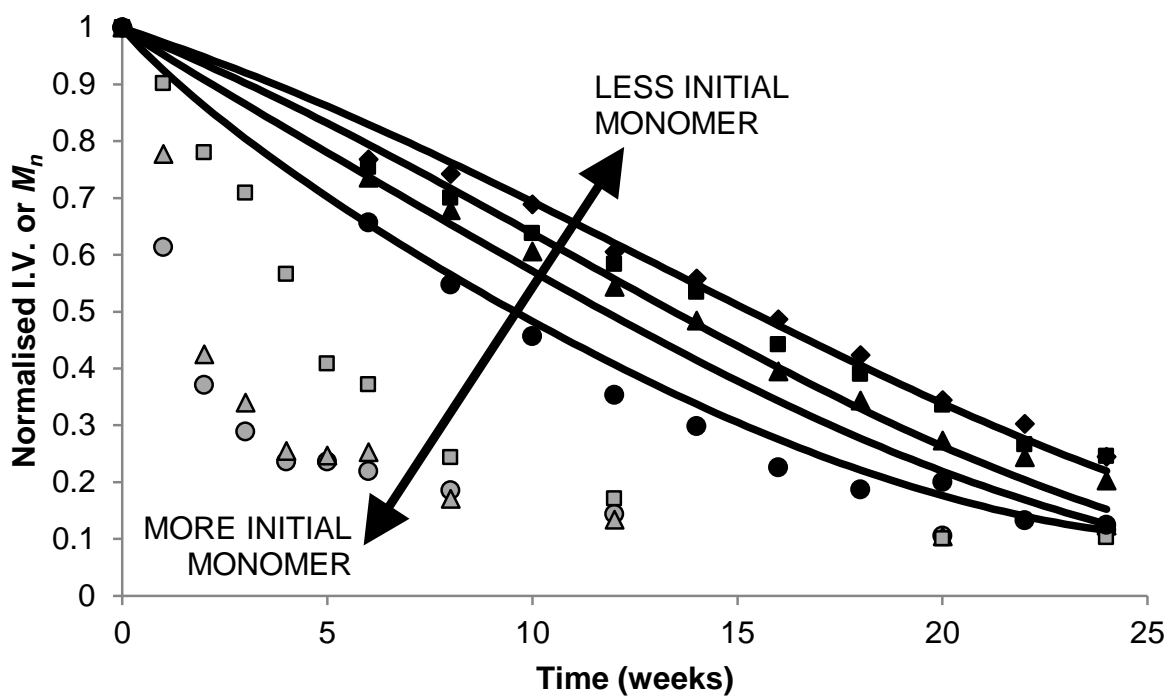

Fig. 8. Alternative presentation for the fitting shown in Fig. 7. Additional experimental data by Paakinaho et al. [12] is included for three samples with higher initial monomer contents. Initial monomer weight percentages are $0.03 \%$ (black diamond), $0.05 \%$ (black square), $0.1 \%$ (black triangle), 0.2\% (black circle), 2\% (grey square), 3\% (grey triangle), and 4\% (grey circle).

Table 3. Parameters used by the model in the data fitting

\begin{tabular}{llllll}
\hline Parameter & Units & Value & Parameter & Units & Value \\
\hline$C_{e 0}$ & $\mathrm{~mol} \mathrm{~m}^{-3}$ & $1.73 \times 10^{4}$ & $M_{0}$ & $\mathrm{~g} \mathrm{~mol}^{-1}$ & 72 \\
$D_{0}$ & $\mathrm{~m}^{2} \mathrm{day}^{-1}$ & $2 \times 10^{-9}$ & $\omega$ & $\mathrm{mol} \mathrm{m}^{-3}$ & $1.73 \times 10^{4}$ \\
$D_{\text {pore }}$ & $\mathrm{m}^{2} \mathrm{day}^{-1}$ & $2 \times 10^{-5}$ & $X_{\max }$ & - & 0.45 \\
$k_{r 2}$ & {$\left[\mathrm{~mol}^{-1} \mathrm{~m}^{3}\right]^{0.5} \mathrm{day}^{-1}$} & $3.4 \times 10^{-6}$ & $p_{x}$ & - & $2 \times 10^{-4}$ \\
$k_{e 2}$ & {$\left[\mathrm{~mol}^{-1} \mathrm{~m}^{3}\right]^{0.5} \mathrm{day}^{-1}$} & $300 * k_{r 1}$ & $V_{c}$ & $\mathrm{~m}^{3}$ & $4.19 \times 10^{-4}$ \\
$k_{e 1}, k_{r 1}$ & day $^{-1}$ & 0 & $\eta_{A}$ & $\mathrm{~mol}^{-1}$ & $6.02 \times 10^{23}$ \\
\hline
\end{tabular}

\section{Conclusions}

The mathematical model developed in part 1 [7], was used to analyse the effect of initial molecular weight. Various hydrolysis mechanisms were considered including noncatalytic random scission, autocatalytic random scission, noncatalytic end scission and autocatalytic end scission. It was found that an increase in initial molecular weight resulted in a decreased molecular weight half-life in the case of random scission. The decrease was greater for noncatalytic hydrolysis than autocatalytic hydrolysis. In contrast for end scission, increasing initial molecular weight resulted in an increased molecular weight half-life. The time taken 
for significant mass loss increased as initial molecular weight increased if mass loss was attributed to end scission. However, mass loss due to random scission was unaffected by initial molecular weight. The model was able to fit experimental data for molecular weight reduction and mass loss for samples with different initial molecular weights [2, 3]. For the most common combination of hydrolysis mechanisms identified in part 1 [7], autocatalytic random scission and autocatalytic end scission, initial molecular weight did not typically affect the molecular weight half-life, although the rate of chain cleavage was affected.

The effect of residual monomer on the degradation was also analysed. For autocatalytic hydrolysis, an inverse square root law was found to relate molecular weight half-life to initial molecular weight. This trend was also identified in experimental data [12] for degradation of samples with different residual monomer contents. The model was able to fit this experimental data by only varying the initial monomer content between fittings. It was found that experimental results for samples with high concentrations of residual monomer may demonstrate similar trends to those expected for noncatalytic hydrolysis.

\section{Acknowledgements}

Andrew Gleadall acknowledges an EPSRC PhD studentship. The authors would also like to acknowledge Kaarlo Paakinaho and Minna Kellomäki from Tampere University of Technology for supplying experimental data from a previous study [12].

\section{References}

[1] Migliaresi C, Fambri L, Cohn D. A study on the in vitro degradation of poly(lactic acid). Journal of Biomaterials Science, Polymer Edition 1994;5:591-606.

[2] Raman C, Berkland C, Kim K, Pack DW. Modeling small-molecule release from PLG microspheres: effects of polymer degradation and nonuniform drug distribution. Journal of Controlled Release 2005;103:149-58.

[3] Pitt GG, Gratzl MM, Kimmel GL, Surles J, Sohindler A. Aliphatic polyesters II. The degradation of poly (DL-lactide), poly ( $\varepsilon$-caprolactone), and their copolymers in vivo. Biomaterials 1981;2:215-20.

[4] Pistner H, Bendi DR, Mühling J, Reuther JF. Poly (l-lactide): a long-term degradation study in vivo: Part III. Analytical characterization. Biomaterials 1993;14:291-8.

[5] Park TG. Degradation of poly(d,l-lactic acid) microspheres: effect of molecular weight. Journal of Controlled Release 1994;30:161-73. 
[6] Huttunen M. Analysis of the factors affecting the inherent viscosity of oriented polylactides during hydrolytic degradation. Journal of Materials Science: Materials in Medicine 2013:1-14.

[7] Gleadall A, Pan J, Kruft M-A. Degradation mechanisms of bioresorbable polyesters, part 1: effects of random scission, end scission and autocatalysis. Acta Biomaterialia.

[8] Wang Y, Pan J, Han X, Sinka C, Ding L. A phenomenological model for the degradation of biodegradable polymers. Biomaterials 2008;29:3393-401.

[9] Han X, Pan J. A model for simultaneous crystallisation and biodegradation of biodegradable polymers. Biomaterials 2009;30:423-30.

[10] Han X, Pan J, Buchanan F, Weir N, Farrar D. Analysis of degradation data of poly(llactide-co-l,d-lactide) and poly(l-lactide) obtained at elevated and physiological temperatures using mathematical models. Acta Biomaterialia 2010;6:3882-9.

[11] Gleadall A, Pan J, Atkinson H. A simplified theory of crystallisation induced by polymer chain scissions for biodegradable polyesters. Polymer Degradation and Stability 2012;97:1616-20.

[12] Paakinaho K, Heino H, Väisänen J, Törmälä P, Kellomäki M. Effects of lactide monomer on the hydrolytic degradation of poly(lactide-co-glycolide) 85L/15G. Journal of the Mechanical Behavior of Biomedical Materials 2011;4:1283-90.

[13] Ellä V, Nikkola L, Kellomäki M. Process-induced monomer on a medical-grade polymer and its effect on short-term hydrolytic degradation. Journal of Applied Polymer Science 2011;119:2996-3003.

[14] Hyon SH, Jamshidi K, Ikada Y. Effects of residual monomer on the degradation of DLlactide polymer. Polymer International 1998;46:196-202.

[15] Tsuji H, Mizuno A, Ikada Y. Properties and morphology of poly(L-lactide). III. Effects of initial crystallinity on long-term in vitro hydrolysis of high molecular weight poly(Llactide) film in phosphate-buffered solution. Journal of Applied Polymer Science 2000;77:1452-64.

[16] Weir N, Buchanan F, Orr J, Farrar D, Dickson G. Degradation of poly-L-lactide. Part 2: increased temperature accelerated degradation. Proceedings of the Institution of Mechanical Engineers, Part H: Journal of Engineering in Medicine 2004;218:321-30.

[17] Tsuji H. In vitro hydrolysis of blends from enantiomeric poly(lactide)s. Part 1. Wellstereo-complexed blend and non-blended films. Polymer 2000;41:3621-30.

[18] Tsuji H. In vitro hydrolysis of blends from enantiomeric poly(lactide)s. Part 4: wellhomo-crystallized blend and nonblended films. Biomaterials 2003;24:537-47. 
[19] Witschi C, Doelker E. Influence of the microencapsulation method and peptide loading on poly(lactic acid) and poly(lactic-co-glycolic acid) degradation during in vitro testing. Journal of Controlled Release 1998;51:327-41.

[20] Li SM, Garreau H, Vert M. Structure-property relationships in the case of the degradation of massive aliphatic poly-( $\alpha$-hydroxy acids) in aqueous media. Journal of Materials Science: Materials in Medicine 1990;1:123-30.

[21] Grizzi I, Garreau H, Li S, Vert M. Hydrolytic degradation of devices based on poly(dllactic acid) size-dependence. Biomaterials 1995;16:305-11.

[22] Paakinaho K, Ellä V, Syrjälä S, Kellomäki M. Melt spinning of poly(l/d)lactide 96/4: Effects of molecular weight and melt processing on hydrolytic degradation. Polymer Degradation and Stability 2009;94:438-42. 